\title{
Science communication scholars use more and more segmentation analyses: Can we take them to the next level?
}

Füchslin, Tobias

\begin{abstract}
Science communication scholars are publishing more and more segmentation analyses as they further our understanding of different audiences and their characteristics. They follow different aims, are therefore difficult to compare and do not lend themselves to more generalisable and theoretical knowledge production. Our field has the potential to follow a demand for more systematic efforts by taking advantage of our high-quality representative data sets focusing on public perceptions of science. Beforehand, however, science communication scholars using segmentation analyses have to identify common goals and overcome a number of hurdles concerning variable selection, methodological approaches, and transparency. Ultimately, a collaborative and systematic application of segmentation analyses could result in truly relevant insights for our field.
\end{abstract}

DOI: https://doi.org/10.1177/0963662519850086

Posted at the Zurich Open Repository and Archive, University of Zurich

ZORA URL: https://doi.org/10.5167/uzh-172697

Journal Article

Accepted Version

Originally published at:

Füchslin, Tobias (2019). Science communication scholars use more and more segmentation analyses: Can we take them to the next level? Public Understanding of Science, 28(7):854-864.

DOI: https://doi.org/10.1177/0963662519850086 
Science communication scholars use more and more segmentation analyses. Can we take them to the next level?

Author

Tobias Füchslin, University of Zürich, Switzerland

ORCID iD

Tobias Füchslin https://orcid.org/0000-0002-2395-9674

Corresponding Author

Tobias Füchslin, Department of Communication and Media Research (IKMZ), University of Zürich, Andreasstr. 15, CH-8050 Zürich, / +41 (0)44 63520 46, t.fuechslin@ikmz.uzh.ch

\section{Abstract}

Science communication scholars are publishing more and more segmentation analyses as they further our understanding of different audiences and their characteristics. They follow different aims, are therefore difficult to compare and do not lend themselves to more generalizable and theoretical knowledge production. Our field has the potential to follow a demand for more systematic efforts by taking advantage of our high-quality representative data sets focusing on public perceptions of science. Beforehand, however, science communication scholars using segmentation analyses have to identify common goals and overcome a number of hurdles concerning variable selection, methodological approaches, and transparency. Ultimately, a collaborative and systematic application of segmentation analyses could result in truly relevant insights for our field.

Keywords

segmentation, cluster analysis, science communication, public understanding of science, survey research

\section{Acknowledgements}

I would like to thank the reviewers and, most of all, Prof. Dr. Mike S. Schäfer for their feedback on this article.

\section{Author Biography}

Tobias Füchslin MA is a research and teaching associate at the division for science communication at the Department of Communication and Media Research (IKMZ), University of Zürich. His research focuses on attitudes towards science and research, scientific literacy, and citizen science. 


\section{Science communication scholars use more and more segmentation} analyses. Can we take them to the next level?

Science communication has developed an appetite for segmentation analyses. For a long time, scholars predominantly analysed the wealth of nationally representative datasets on people's perceptions of, attitudes towards and knowledge of science through multivariate analyses such as linear regression models (Bauer, 2009). They give insights into variable relationships but do not further our understanding of different audiences of science communication and their characteristics. That is why our field needs segmentation analyses - a form of explorative data analysis that divides a population that is diverse in analytically relevant characteristics into relatively homogenous, yet mutually exclusive subgroups (Metag \& Schäfer, 2018). This journal published a first peer-reviewed segmentation analysis of nationally representative survey data in the field of science communication, outlining that the Japanese population can be interpreted as four distinct and relatively homogenous segments called "Inquisitive", "Sciencephiles", "Life-centered", and "Low-interest" (Kawamoto, Nakayama, \& Saijo, 2011). Before, similar analyses were only found in non-academic reports, such as a series of segmentations of the UK population (e.g., Ipsos MORI, 2011; OST \& Wellcome Trust, 2000). Since then, however, more and more segmentation analyses were published in research journals, such as Public Understanding of Science (PUS), Environmental Communication and the Journal of Science Communication. Just recently, PUS was part of a burst of segmentations starting in 2016, including analyses for South Africa (Guenther \& Weingart, 2017), Switzerland (Schäfer, Füchslin, Metag, Kristiansen, \& Rauchfleisch, 2018), Japan (Okamura, 2016), as well as China, South Korea, China, and the USA (Pullman, Chen, Zou, Hives, \& Liu, 2018). Complementing this burst, a special issue on "Audience Segments in Environmental and Science Communication" was published in Environmental Communication (Metag \& Schäfer, 2018).

It is noticeable that these segmentations (cf. Table 1) mostly follow different aims, are therefore difficult to compare and do not lend themselves easily to generalisation ${ }^{1}$. Most of these studies do not aim to build a body of systematic knowledge but have more practical aims and, therefore, are not motivated by a common set of goals and are also less theoretically-driven. Some studies heavily focus on country comparisons (Pullman et al., 2018), some on temporal developments within the same country (Okamura, 2016), whereas others aim to improve science communication efforts in general (Schäfer et al., 2018), to recruit potential citizen scientists (Füchslin, Schäfer, \& Metag, 2019), to increase people's scientific literacy (Kawamoto et al., 2011), or by offering efficient "post-hoc" segmentations (Runge, Brossard, \& Xenos, 2018). Against this backdrop, Scheufele (2018) recently demanded that fields like science and environmental communication strive for more systematic segmentation efforts, taking into account differences between issues, issue cycles, cultural or national contexts, and methodological approaches. He suggests that such efforts would be valuable for basic social science research and not only for specific communication purposes.

\footnotetext{
${ }^{1}$ An exception would be the reoccurrence of groups like the "Sciencephiles" or the "Disengaged/Disinterested, whereas the remaining groups seem to be more varied across countries and contexts.
} 
Science communication research is ideally suited to realise such systematic efforts: First, science communication is still in the early phase of employing segmentation analyses. This allows us to think about systematic efforts before different research terms are set in their incomparable ways. Second, our field has established many nationally representative surveys like the annual "Science and Engineering Indicators" in the US, the Eurobarometer or the "Wissenschaftsbarometer" in Germany or Switzerland (for an overview, cf. Bauer \& Falade, 2014). This means that researchers can draw on a lot of high-quality survey data, some of them available for several countries and cross-national comparative analysis, and some of them available over long periods of time, partly for decades. Third, most of these surveys already have substantial topical overlap. Almost all of them assess people's attitudes towards, knowledge of, and perceptions of science and often share measurements of a handful of key theoretical dimensions (cf. Bauer, 2009; Besley, 2013). While there remain crucial differences between these surveys, - some ask about "science \& technology", others about "science and research" - the overlap is big enough to identify a common topic like "public perceptions of science".

This creates a situation where researchers have high quality data, can focus on a common topic and start using segmentation analyses by systematically varying national and temporal contexts. Segmentation analyses in climate change communication have already shown that proposed segmentation solutions of one country can be directly applied to another country (M. Morrison, Duncan, Sherley, \& Parton, 2013), or tracked across time within the same country (Mark Morrison, Parton, \& Hine, 2018). Science communication could mimic and even surpass such efforts.

Beforehand, however, segmentation analyses in science communication have to overcome a number of hurdles. I will outline why and how we need to a) streamline variable selection and measurement, b) focus on methodological approaches that favour robust solutions, and c) improve transparency and facilitate continued efforts. I make my points by focusing on prior segmentation analyses in science communication (or related fields) that work with representative datasets exclusively (cf. Table 1).

We need to streamline variable selection and measurement

Ideally, a clear theoretical framework guides variable selection in any systematic approach to segmentation analyses. But Hine et al. (2014) observed many "atheoretical" approaches to variable selection in climate change communication segmentations. Analyses in science communication are mostly isolated undertakings, minimising researchers' need and effort to develop a theoretical framework. As a result, analyses show differences on at least four levels. As Metag and Schäfer (2018) point out, a first level of differences pertains to whether researchers segment along psychographic, sociodemographic, or behavioural variables. Segmentations in science communication are quite similar in that they mostly employ a psychographic approach focusing on attitudes towards science (and technology/research). Some studies, however, go beyond these variables and add sociodemographics (Besley, 2018), behavioural variables like media consumption (Kawamoto et al., 2011), or both (Guenther \& Weingart, 2017). A second level of differences appears in the number and selection of the- 
oretical dimensions. For example, Pullman et al. (2018) focus on the single dimension of general attitudes towards science, while other studies conceptualise attitudes towards science through as many as five dimensions, covering cognitive, affective, conative attitudes towards science, as well as reservations and hopes towards science, subjective norms regarding science and society, and informational behaviour (Schäfer et al., 2018). Other studies use different dimensions altogether and look at more specific constructs such as deference to scientific authority (Runge et al., 2018) or attitudes towards science policy making (Okamura, 2016). Further studies started with broadly defined dimensions, applied factor analysis to all their items and subsequently described new emerging dimensions such as "scepticism about science careers" or "perceived independence of science and scientists" (Castell et al., 2014). As a third level, the number of items representing a certain dimension also varies considerably. One example is the recurring dimension of "hopes and reservations regarding science" where Besley (2018) uses three items while (Schäfer et al., 2018) use seven. A more extreme example is the generic category of "attitudes towards science", represented by six (Pullman et al., 2018) up to 19 items (Kawamoto et al., 2011). Lastly, a fourth level pertains to the differences in variable measurement, both in wording and scale. This leads to cases where three different studies assess whether people agree that "science improves our lives" by applying four-, five, or ten-point scales, respectively, and using three (albeit slightly) different wordings (Nisbet \& Markowitz, 2014; Pullman et al., 2018; Schäfer et al., 2018).

While there is considerable heterogeneity in variable selection, the differences are smaller than they appear. Some authors label very similar items with different dimensions or assign them to a broad category like "attitudes towards science". Many prior analyses could have followed the already mentioned categorisation proposed by Schäfer et al. (2018): Pullman et al. (2018) would have covered hopes and reservations towards science as well as the subjective norm regarding informational behaviour. Besley (2018) included items that covered hopes and reservations as well as the cognitive dimension of attitudes towards science and technology. The problem is that segmentation analyses do not incentivise to improve conceptual clarity and comparability. In both examples, it would not have mattered to which theoretical dimension researchers would have assigned their items as they ended up analysing all items together.

I chose the categories by Schäfer et al. (2018) for illustrative purposes only. Future research should explore the most theoretically useful and practical common ground across established science communication surveys. This most certainly means that the set of variables has to be on the smaller side. Technical analyses in other fields have shown that some segment-solutions can be replicated by using fewer variables by focusing on the most powerful predictors (Chryst et al., 2018). Füchslin, Schäfer, and Metag (2018) replicated the solution by Schäfer et al. (2018) using 10 rather than 20 items. Efforts could also clarify further questions regarding the inclusion of sociodemographic and behavioural variables in the final framework or regarding the issue of items focusing on attitudes towards "science and technology" versus "science and research". 
Getting to a point of having identical items with identical measurements or even having new standardised scales seems a bit too optimistic at this point. If anything, it seems more plausible that established surveys would add new items than alter their existing item measurements. In their review of segmentations in climate change communication, Hine et al. (2014) point out that agreeing on common dimensions would be a step in the right direction.

I think that science communication, ideally, would identify a compact and widely applicable theoretical framework, maybe even find and promote a small but common set of standard items across nationally representative surveys. Ideally, such a framework would define a clear context such as "public attitudes towards science and research" and provide a causal model describing relations between the included core constructs, similar to what we see in related fields like risk communication (van der Linden, 2015). This would benefit all scholars interested in segmentation research, because it would not only help to design research, but would also incentivise surveys - new or even more established ones - to measure variables that cater to the proposed theoretical model. However, our field will only find the motivation to develop a framework if the goals of segmentations move away from serving specific communication purposes to investigating more systematic questions. One of the best ways to unify behind a more substantial research question seem to be collaborations between research teams something which should be more easily achievable in a relatively small community like science communication. 
Table 1: Overview of representative segmentation analyses in science communication

\begin{tabular}{|c|c|c|c|c|c|c|c|c|}
\hline Study & Topical Focus & $\begin{array}{l}\text { Aim of Segmentation } \\
\text { (additional analyses are } \\
\text { not considered) }\end{array}$ & Sample & Number of Items & Method & $\begin{array}{l}\text { Robustness } \\
\text { checks? }\end{array}$ & Results & Directly Related Studies \\
\hline $\begin{array}{l}\text { Füchslin et } \\
\text { al. (2019) }\end{array}$ & $\begin{array}{l}\text { Interest to } \\
\text { participate in } \\
\text { citizen sci- } \\
\text { ence projects }\end{array}$ & $\begin{array}{l}\text { Reconstructing target } \\
\text { groups for citizen sci- } \\
\text { ence projects; improving } \\
\text { communication of re- } \\
\text { cruitment }\end{array}$ & $\begin{array}{l}\text { National repre- } \\
\text { sentative survey, } \\
\text { subset }(N=381) \\
\text { Switzerland, } 2016\end{array}$ & $\begin{array}{l}13 \text { items (dichotomous and } \\
5 \text {-point) covering de- } \\
\text { mographics and attitudes to- } \\
\text { wards science and research }\end{array}$ & $\begin{array}{l}\text { Latent Class } \\
\text { Analysis }\end{array}$ & $\begin{array}{l}\text { Within method } \\
\text { statistical ro- } \\
\text { bustness } \\
\text { Not across } \\
\text { methods }\end{array}$ & $\begin{array}{l}\text { Five segments: "Free-Timers", "Senior } \\
\text { Sciencephiles", "Young Science- } \\
\text { philes", "Intrigued Adolescents", } \\
\text { "Fully Employed Parents" }\end{array}$ & - \\
\hline $\begin{array}{l}\text { Pullman et } \\
\text { al. (2018) }\end{array}$ & $\begin{array}{l}\text { Attitudes to- } \\
\text { wards Sci- } \\
\text { ence \& Tech- } \\
\text { nology }\end{array}$ & $\begin{array}{l}\text { Contribute to interna- } \\
\text { tional comparative re- } \\
\text { search }\end{array}$ & $\begin{array}{l}\text { World Value Survey, } \\
\text { sixth wave: USA } \\
(N=2159) \text {, China } \\
(N=1580) \text {, South Ko- } \\
\text { rea }(N=1145) \text {, Japan } \\
(N=1847)\end{array}$ & $\begin{array}{l}6 \text { items (10-point) covering } \\
\text { general attitudes towards } \\
\text { science \& technology }\end{array}$ & $\begin{array}{l}\text { Latent Class } \\
\text { Analysis (lim- } \\
\text { ited to } 5 \text { clas- } \\
\text { ses) }\end{array}$ & Not reported & $\begin{array}{l}\text { Five recurring segments: "High-posi- } \\
\text { tive”, “Moderate”, "Negative”, “Mod- } \\
\text { erate-Positive”, “Negative \& Positive” } \\
\text { At least two segments represented in } \\
\text { each country }\end{array}$ & - \\
\hline $\begin{array}{l}\text { Runge et al. } \\
\text { (2018) }\end{array}$ & $\begin{array}{l}\text { Science com- } \\
\text { munication }\end{array}$ & $\begin{array}{l}\text { Post-hoc creation of } \\
\text { segments for science } \\
\text { communication re- } \\
\text { searchers and practition- } \\
\text { ers }\end{array}$ & $\begin{array}{l}\text { National repre- } \\
\text { sentative survey } \\
(\mathrm{N}=2858) \\
\text { USA, 2014 }\end{array}$ & $\begin{array}{l}5 \text { items (7- or 11-point) cov- } \\
\text { ering ideologies and atti- } \\
\text { tudes to scientists }\end{array}$ & $\begin{array}{l}\text { Hierarchical } \\
\text { clustering }\end{array}$ & $\begin{array}{l}\text { Within method } \\
\text { statistical ro- } \\
\text { bustness } \\
\text { Not across } \\
\text { methods }\end{array}$ & $\begin{array}{l}\text { Five segments: "Protective progres- } \\
\text { sives", “Engaged moderates", "Main- } \\
\text { stream traditionalists", "Disengaged } \\
\text { moderates", "Distrustful traditional- } \\
\text { ists" }\end{array}$ & - \\
\hline $\begin{array}{l}\text { Besley } \\
(2018)\end{array}$ & $\begin{array}{l}\text { Views about } \\
\text { science and } \\
\text { technology }\end{array}$ & $\begin{array}{l}\text { Trying to understand } \\
\text { views about science and } \\
\text { technology using seg- } \\
\text { mentation }\end{array}$ & $\begin{array}{l}\text { National repre- } \\
\text { sentative survey } \\
(\mathrm{N}=1266) \\
\text { USA, 2016 }\end{array}$ & $\begin{array}{l}11 \text { items (wide range of } \\
\text { scales) covering de- } \\
\text { mographics, ideology, atti- } \\
\text { tudes towards science and } \\
\text { technology }\end{array}$ & $\begin{array}{l}\text { Latent profile } \\
\text { analysis }\end{array}$ & Not reported & $\begin{array}{l}\text { Six segments: “Disengaged", "Wor- } \\
\text { ried”, Cautious Conservatives", “Mod- } \\
\text { erate Optimists", "Liberal Science- } \\
\text { philes", “Conservative Sciencephiles" }\end{array}$ & - \\
\hline $\begin{array}{l}\text { Schäfer et } \\
\text { al. (2018) }\end{array}$ & $\begin{array}{l}\text { Attitudes to- } \\
\text { wards science } \\
\text { and research }\end{array}$ & $\begin{array}{l}\text { Reconstructing audi- } \\
\text { ences of science com- } \\
\text { munication; improving } \\
\text { science communication }\end{array}$ & $\begin{array}{l}\text { National repre- } \\
\text { sentative survey } \\
\text { ( } \mathrm{N}=1051 \text { ) } \\
\text { Switzerland, } 2016\end{array}$ & $\begin{array}{l}20 \text { items (5-point) covering } \\
\text { attitudes towards science } \\
\text { and research }\end{array}$ & $\begin{array}{l}\text { Latent Class } \\
\text { Analysis }\end{array}$ & $\begin{array}{l}\text { Compared to } \\
\text { factor analysis } \\
\text { plus hierar- } \\
\text { chical cluster } \\
\text { analysis. }\end{array}$ & $\begin{array}{l}\text { Four segments: "Sciencephiles", “Crit- } \\
\text { ically Interested”, "Passive Support- } \\
\text { ers", "Disengaged” }\end{array}$ & $\begin{array}{l}\text { Short scale development } \\
\text { (Füchslin et al., 2018) }\end{array}$ \\
\hline $\begin{array}{l}\text { Cámara, } \\
\text { van den } \\
\text { Muñoz } \\
\text { Eynde, and } \\
\text { López Ce- } \\
\text { rezo (2018) }\end{array}$ & $\begin{array}{l}\text { Public per- } \\
\text { ception of sci- } \\
\text { ence \& tech- } \\
\text { nology }\end{array}$ & $\begin{array}{l}\text { Providing evidence of } \\
\text { the group of the "Critical } \\
\text { Engagers" }\end{array}$ & $\begin{array}{l}\text { National repre- } \\
\text { sentative survey } \\
(\mathrm{N}=6354) \\
\text { Spain, } 2014\end{array}$ & $\begin{array}{l}4 \text { items (3-/5-point) covering } \\
\text { support of and optimism to- } \\
\text { wards science }\end{array}$ & $\begin{array}{l}\text { Manual attribu- } \\
\text { tion }\end{array}$ & N.A. & $\begin{array}{l}\text { Two segments: "Critical Engagers" } \\
\text { and "Others" }\end{array}$ & - \\
\hline $\begin{array}{l}\text { Guenther } \\
\text { and } \\
\text { Weingart } \\
\text { (2017) }\end{array}$ & $\begin{array}{l}\text { Attitudes to- } \\
\text { wards science } \\
\& \text { technology }\end{array}$ & $\begin{array}{l}\text { Investigating cultural } \\
\text { context of attitudes to- } \\
\text { wards science and tech- } \\
\text { nology }\end{array}$ & $\begin{array}{l}\text { National repre- } \\
\text { sentative survey } \\
(\mathrm{N}=3183) \\
\text { South Africa, } 2010\end{array}$ & $\begin{array}{l}30 \text { items (dichotomous) cov- } \\
\text { ering sociodemographics, } \\
\text { science information sources } \\
\text { and scientific literacy }\end{array}$ & $\begin{array}{l}\text { Hierarchical } \\
\text { cluster analysis }\end{array}$ & Not reported & Six sociodemographic segments & $\begin{array}{l}\text { Qualitative follow-up } \\
\text { (Guenther, Weingart, \& } \\
\text { Meyer, 2018) }\end{array}$ \\
\hline
\end{tabular}




\begin{tabular}{|c|c|c|c|c|c|c|c|c|}
\hline $\begin{array}{l}\text { Okamura } \\
\text { (2016) }\end{array}$ & $\begin{array}{l}\text { Attitudes to- } \\
\text { wards Sci- } \\
\text { ence \& Tech- } \\
\text { nology policy- } \\
\text { making }\end{array}$ & $\begin{array}{l}\text { Enhancement of under- } \\
\text { standing of audiences } \\
\text { regarding science \& } \\
\text { technology policymak- } \\
\text { ing; temporal compari- } \\
\text { sons }\end{array}$ & $\begin{array}{l}\text { National repre- } \\
\text { sentative survey } \\
(\mathrm{N}=6136) \\
\text { Japan, } 2011\end{array}$ & $\begin{array}{l}3 \text { items (4-point) regarding } \\
\text { attitudes towards science \& } \\
\text { technology policy }\end{array}$ & $\begin{array}{l}\text { K-means cluster } \\
\text { analysis to re- } \\
\text { construct three } \\
\text { conceptualised } \\
\text { segments }\end{array}$ & $\begin{array}{l}\text { Clusters re- } \\
\text { mained stable } \\
\text { over } 8 \text { months } \\
\text { (eight waves) }\end{array}$ & $\begin{array}{l}\text { Three segments: "Attentive", "Inter- } \\
\text { ested", "Residual” }\end{array}$ & - \\
\hline $\begin{array}{l}\text { Castell et } \\
\text { al. (2014) }\end{array}$ & $\begin{array}{l}\text { Attitudes to- } \\
\text { wards science } \\
\text { \& technology }\end{array}$ & $\begin{array}{l}\text { Continued reporting on } \\
\text { attitudes towards to sci- } \\
\text { ence }\end{array}$ & $\begin{array}{l}\text { National repre- } \\
\text { sentative survey } \\
\text { (N=1749) } \\
\text { UK, } 2013\end{array}$ & $\begin{array}{l}77 \text { items (5-point) covering } \\
\text { general attitudes towards } \\
\text { science \& technology } \\
\text { Condensed into } 15 \text { factors } \\
\text { representing } 76 \text { items }\end{array}$ & $\begin{array}{l}\text { Factor analysis } \\
\text { k-means cluster } \\
\text { analysis }\end{array}$ & Not reported & $\begin{array}{l}\text { Six Segments: “Late Adopters", "Con- } \\
\text { cerned", "Disengaged Sceptics", "In- } \\
\text { different", "Distrustful Engagers", } \\
\text { "Confident Engagers" }\end{array}$ & $\begin{array}{l}\text { Predecessor UK segmenta- } \\
\text { tions (Ipsos MORI, 2011; } \\
\text { MORI, 2005; OST \& Well- } \\
\text { come Trust, 2000; Re- } \\
\text { search Councils UK, 2008) }\end{array}$ \\
\hline $\begin{array}{l}\text { Nisbet and } \\
\text { Markowitz } \\
\text { (2014) }\end{array}$ & $\begin{array}{l}\text { Beliefs about } \\
\text { science and } \\
\text { society }\end{array}$ & $\begin{array}{l}\text { Investigating influence } \\
\text { of beliefs about science } \\
\text { and society on public } \\
\text { opinion about stem cell } \\
\text { research }\end{array}$ & $\begin{array}{l}\text { Eight waves of na- } \\
\text { tional representa- } \\
\text { tive surveys } \\
\text { (N=8105) } \\
\text { USA, 2002-2010 } \\
\text { (not 2009) }\end{array}$ & $\begin{array}{l}4 \text { items (4-point) covering } \\
\text { hopes and reservations to- } \\
\text { wards science } \\
\text { condensed into two factors }\end{array}$ & $\begin{array}{l}\text { Manual attribu- } \\
\text { tion }\end{array}$ & N.A. & $\begin{array}{l}\text { Four segments: "Scientific Optimists", } \\
\text { "Scientific Pessimists", "Conflicted", } \\
\text { "Disengaged" }\end{array}$ & - \\
\hline $\begin{array}{l}\text { Ipsos MORI } \\
\text { (2013) }\end{array}$ & $\begin{array}{l}\text { Attitudes to- } \\
\text { wards science } \\
\text { \& technology }\end{array}$ & $\begin{array}{l}\text { Commissioned report: } \\
\text { explore current atti- } \\
\text { tudes towards science } \\
\text { and technology and bio- } \\
\text { technology }\end{array}$ & $\begin{array}{l}\text { National repre- } \\
\text { sentative survey } \\
(\mathrm{N}=2000) \\
\text { Australia, } 2012\end{array}$ & $\begin{array}{l}14 \text { items (11-point) covering } \\
\text { general attitudes towards } \\
\text { science \& technology }\end{array}$ & $\begin{array}{l}\text { K-means cluster } \\
\text { analysis }\end{array}$ & Not reported & Four unnamed segments & - \\
\hline $\begin{array}{l}\text { Hurtado } \\
\text { and Cerezo } \\
\text { (2012) }\end{array}$ & $\begin{array}{l}\text { Public per- } \\
\text { ception of sci- } \\
\text { ence \& tech- } \\
\text { nology }\end{array}$ & $\begin{array}{l}\text { General analysis of sci- } \\
\text { entific culture }\end{array}$ & $\begin{array}{l}\text { Pseudo-representa- } \\
\text { tive sample } \\
\text { (N=7739) } \\
\text { Region of Ibero- } \\
\text { America, } 2007\end{array}$ & $\begin{array}{l}11 \text { items (3-/4-point) cover- } \\
\text { ing attitudes towards science } \\
\text { and technology }\end{array}$ & $\begin{array}{l}\text { Hierarchical } \\
\text { cluster analysis }\end{array}$ & Not reported & Three unnamed segments & - \\
\hline $\begin{array}{l}\text { Kawamoto } \\
\text { et al. } \\
\text { (2011) }\end{array}$ & $\begin{array}{l}\text { Science Com- } \\
\text { munication } \\
\text { Scientific lit- } \\
\text { eracy }\end{array}$ & $\begin{array}{l}\text { Improving science com- } \\
\text { munication to enhance } \\
\text { scientific literacy }\end{array}$ & $\begin{array}{l}\text { National repre- } \\
\text { sentative survey } \\
(\mathrm{N}=1286) \\
\text { Japan, } 2008\end{array}$ & $\begin{array}{l}65 \text { items covering social, lit- } \\
\text { eracy and science questions } \\
\text { Condensed into } 3 \text { factors } \\
\text { representing } 38 \text { items }\end{array}$ & $\begin{array}{l}\text { Factor Analysis } \\
\text { K-means cluster } \\
\text { analysis }\end{array}$ & Not reported & $\begin{array}{l}\text { Four segments: "Inquisitive”, "Scien- } \\
\text { cephiles", "Life-centered", “Low-in- } \\
\text { terest” }\end{array}$ & $\begin{array}{l}\text { Representation of Seg- } \\
\text { ments at science café } \\
\text { events (Kawamoto, Naka- } \\
\text { yama, \& Saijo, 2013) }\end{array}$ \\
\hline $\begin{array}{l}\text { Sweeney } \\
\text { Research } \\
\text { (2011) }\end{array}$ & $\begin{array}{l}\text { Attitudes to- } \\
\text { wards and } \\
\text { engagement } \\
\text { with science } \\
\text { and technol- } \\
\text { ogy }\end{array}$ & $\begin{array}{l}\text { Tracking community at- } \\
\text { titudes to and engage- } \\
\text { ment with science }\end{array}$ & $\begin{array}{l}\text { Community repre- } \\
\text { sentative survey } \\
(\mathrm{N}=800) \\
\text { Victoria, AUS, } 2011\end{array}$ & $\begin{array}{l}3 \text { items (scale unclear) cover- } \\
\text { ing interest and information } \\
\text { seeking of science and tech- } \\
\text { nology }\end{array}$ & manual & N.A. & Six unnamed segments & $\begin{array}{l}\text { Inaccessible predecessor } \\
\text { segmentation in } 2007 \\
\text { Focus group study with un- } \\
\text { interested segment (Cor- } \\
\text { mick, 2012) }\end{array}$ \\
\hline
\end{tabular}


We need methodological approaches that favour robust segment solutions

In line with this efforts towards a joint, or at least more explicit and ideally standardized, theoretical framework for variable selection, segmentation analyses in science communication should also aim to strengthen their methodological approach. Current analyses have employed almost all the most common statistical techniques for clustering data, ranging from distanced-based procedures like hierarchical (Runge et al., 2018) and k-means clustering (Ipsos MORI, 2013) to model-based procedures like latent class (Schäfer et al., 2018) and latent profile analysis (Pullman et al., 2018), sometimes with running a factor analysis in a first step (Kawamoto et al., 2011) and sometimes without (Guenther \& Weingart, 2017). Other studies did not employ multivariate statistics at all and applied "manual clustering" by defining which combinations of variable expressions would lead to which kind of segment (Cámara et al., 2018; Nisbet \& Markowitz, 2014; Sweeney Research, 2011).

The variability in methods is related to the selection and measurement of variables. Researchers that had continuous variables often opted for k-means clustering while those with ordinal variables preferred latent class or latent profile analyses. Since this commentary focuses on segmentations based on representative survey data, all studies start with ordinal variable measurements. What leads to continuous variables is the calculation of indices that sometimes struggle with reliability coefficients (e.g., Besley, 2018; Okamura, 2016) or the application of factor analysis to reduce the large number of items. As a downside, these factor analyses often lead to novel dimensions that are hard to interpret as they consist of multiple items with differing and sometimes very low factor loadings. Additionally, missing values tend to result from applying factor analyses to survey data (e.g., Kawamoto et al., 2011).

We do not see the same methodological variability within analyses, however, that we see across studies - leading to another challenge for our research field. Segmentation analyses are explorative procedures allowing the parallel application of multiple methods. Yet, almost none of the referenced analyses have compared their solutions across methods (cf. Table 1: Robustness checks). As a result, it remains unclear how robust these solutions are. This is less of a concern if researchers aim for practical segmentations. It would be less desirable, however, to build systematic approaches on solutions that are largely influenced by the segmentation method.

Future segmentations can advance in two regards: first, we should increase our understanding of segmentation methods when applied to a typical set of science communication variables. At this point, it seems reasonable to focus on procedures like latent class analysis that cater to ordinal variables. This is especially true if researchers in science communication can agree on a small set of items, as this would remove the temptation to reduce the number of variables through factor analysis. Second, researchers should begin to explore cluster solutions across multiple methods. This can go along with expanding the methodological repertoire by including procedures like random forest clustering (Giannella \& Fischer, 2016), fuzzy clustering (Neunhoeffer \& Teubner, 2018) or density-based clustering (Kassambara, 2017). 
We need to focus on transparency and facilitate systematic efforts

All potential improvements in variable selection and methodological approaches are idle if authors do not facilitate the systematic continuation of their proposed segmentations. This goal ultimately hinges on two aspects: transparency and methodology.

Systematic segmentation efforts in science communication require transparency in reporting methods and results. Most of the studies outlined in table 1 did not or only superficially report on details like the statistical software they used (i.e. name of software version or package), the application of survey weights, the treatment and potential imputation of missing values, the rationale for selection of cluster solutions, and "goodness of solutions" indicators (e.g. reporting dendrograms, BIC-values, discriminant analysis, etc.). For example, the series of UK segmentations has produced reports that present item- and segment-descriptions in large detail. But, when it comes to the methodological details, the report merely mentions that the authors ran a factor analysis and then administered a combination of hierarchical and kmeans clustering (e.g., Ipsos MORI, 2011). Readers never get to see the factor loadings or what the dendrograms of the hierarchical solutions looked like.

Ideally, researchers should pick segmentation methods that facilitate transparent reporting and the continuation of prior efforts. For example, the combination of factor analysis and hierarchical clustering is an approach that does not lend itself to transparent reporting. Combining two exploratory methods entails many researcher degrees of freedom that authors simply cannot report on with a handful of statistics. Model-based approaches are inherently easier to report on in terms of choosing the final cluster solution and describing the goodness of the solution through widely used indicators like BIC- and AIC-values. They also offer solutions that build on regression models. This allows researchers to reuse established regression models and assign new cases to predefined segments - opening the door for continued and systematic application and testing of proposed solutions.

If authors used methods that facilitated transparent reporting and continued efforts, future research could take proposed segment solutions and apply them to their data for, say, another country or another time period. In climate change communication, the "Six Americas" solution (Maibach, Leiserowitz, Roser-Renouf, \& Mertz, 2011) highlights this potential; researchers have applied it to other countries and periods (M. Morrison et al., 2013; Mark Morrison et al., 2018) and developed shorter scales (Chryst et al., 2018; Swim \& Geiger, 2015). The continued efforts we see in science communication are be authors taking qualitative looks at specific clusters discovered in their previous studies (Kawamoto et al., 2013).

\section{We can reap the benefits}

Overall, segmentation analyses in science communication are becoming increasingly popular. Because they are not always necessarily working toward a goal of building a body of systematic knowledge, analyses currently feature a lot of variability in variable selection and measurement, application of methods, and facilitation of continued efforts. Our field could take advantage of its high-quality data sets - many are publicly available (e.g., Eurobarometer or 
World Value Survey) or are likely to be made accessible by its owners - with large topical overlap and aim at systematic segmentation efforts, if we reduce and improve upon these variabilities. Luckily, dedicated future research can easily address all these challenges: Improved application of methods and more transparent reporting do not require any new advances but good preparation and mid- to long-term planning. Working out a compact and widely applicable theoretical framework will be more challenging as it requires researchers to collaborate and agree to focus on more theory-driven systematic knowledge production. However, the investment should clearly be worth the effort in this case - finding a conceptually reasonable common ground among data sets could initiate a systematic application of segmentation analyses and result in truly relevant insights for our field.

\section{References}

Bauer, M. W. (2009). The Evolution of Public Understanding of Science-Discourse and Comparative Evidence. Science, Technology and Society, 14(2), 221-240.

https://doi.org/10.1177/097172180901400202

Bauer, M. W., \& Falade, B. A. (2014). Public understanding of science: survey research around the world. In M. Bucchi \& B. Trench (Eds.), Routledge Handbook of Public Communication of Science and Technology: Second edition (pp. 140-159). London: Routledge.

Besley, J. C. (2013). The State of Public Opinion Research on Attitudes and Understanding of Science and Technology. Bulletin of Science, Technology \& Society, 33(1-2), 12-20. https://doi.org/10.1177/0270467613496723

Besley, J. C. (2018). Audiences for Science Communication in the United States. Environmental Communication, 12(8), 1005-1022. https://doi.org/10.1080/17524032.2018.1457067

Cámara, M., van den Muñoz Eynde, A., \& López Cerezo, J. A. (2018). Attitudes towards science among Spanish citizens: The case of critical engagers. Public Understanding of Science (Bristol, England), 27(6), 690-707. https://doi.org/10.1177/0963662517719172

Castell, S., Charlton, A., Clemence, M., Pettigrew, N., Pope, S., Quigley, A., . . Silman, T. (2014). Public Attitudes to Science 2014: Main Report. Retrieved from https://www.ipsos.com/ipsos-mori/en-uk/public-attitudes-science-2014

Chryst, B., Marlon, J., van der Linden, S., Leiserowitz, A., Maibach, Edward, \& Roser-Renouf, C. (2018). Global Warming's "Six Americas Short Survey": Audience Segmentation of Climate Change Views Using a Four Question Instrument. Environmental Communication, 12(8), 1109-1122. https://doi.org/10.1080/17524032.2018.1508047

Cormick, C. (2012). How Do We Gain the Interest of People Who Are Uninterested in Science and Technology? In H. van Lente, C. Coenen, T. Fleischer, K. Konrad, L. Krabbenborg, C. Milburn, ... T. Zülsdorf (Eds.), Studies of New and Emerging Technologies: Vol. 3. Little by Little: Expansions of Nanoscience and Emerging Technologies (pp. 77-88). Amsterdam: IOS Press.

Füchslin, T., Schäfer, M. S., \& Metag, J. (2018). A Short Survey Instrument to Segment Populations According to Their Attitudes Toward Science. Scale Development, Optimization and Assessment. Environmental Communication, 12(8), 1095-1108.

https://doi.org/10.1080/17524032.2018.1461673 
Füchslin, T., Schäfer, M. S., \& Metag, J. (2019). Who wants to be a citizen scientist? Identifying the overall potential of citizen science and target segments in Switzerland. Public Understanding of Science (Bristol, England), accepted.

Giannella, E., \& Fischer, C. S. (2016). An inductive typology of egocentric networks. Social Networks, 47, 15-23. https://doi.org/10.1016/i.socnet.2016.02.003

Guenther, L., \& Weingart, P. (2017). Promises and reservations towards science and technology among South African publics: A culture-sensitive approach. Public Understanding of Science (Bristol, England), 963662517693453.

https://doi.org/10.1177/0963662517693453

Guenther, L., Weingart, P., \& Meyer, C. (2018). "Science is Everywhere, but No One Knows It": Assessing the Cultural Distance to Science of Rural South African Publics. Environmental Communication, 12(8), 1046-1061. https://doi.org/10.1080/17524032.2018.1455724

Hine, D. W., Reser, J. P., Morrison, Mark, Phillips, W. J., Nunn, P., \& Cooksey, R. (2014). Audience segmentation and climate change communication: Conceptual and methodological considerations. Wiley Interdisciplinary Reviews: Climate Change, 5(4), 441-459. https://doi.org/10.1002/wcc.279

Hurtado, M. C., \& Cerezo, J. A. L. (2012). Political dimensions of scientific culture: Highlights from the Ibero-American survey on the social perception of science and scientific culture. Public Understanding of Science, 21(3), 369-384.

https://doi.org/10.1177/0963662510373871

Ipsos MORI. (2011). Public Attitudes to Science 2011: Main Report. Retrieved from https://www.ipsos.com/ipsos-mori/en-uk/public-attitudes-science-2011

Ipsos MORI. (2013). Community Attitudes towards Emerging Technology Issues: Biotechnology.

Kassambara, A. (2017). Practical guide to cluster analysis in R: Unsupervised machine learning (Edition 1). Multivariate Analysis I. [Erscheinungsort nicht ermittelbar]: STHDA.

Kawamoto, S., Nakayama, M., \& Saijo, M. (2011). A survey of scientific literacy to provide a foundation for designing science communication in Japan. Public Understanding of Science (Bristol, England), 22(6), 674-690. https://doi.org/10.1177/0963662511418893

Kawamoto, S., Nakayama, M., \& Saijo, M. (2013). Using a scientific literacy cluster to determine participant attitudes in scientific events in Japan, and potential applications to improving science communication. Journal of Science Communication, 12(01).

https://doi.org/10.22323/2.12010201

Maibach, Edward W., Leiserowitz, A., Roser-Renouf, C., \& Mertz, C. K. (2011). Identifying like-minded audiences for global warming public engagement campaigns: An audience segmentation analysis and tool development. PloS One, 6(3), e17571. https://doi.org/10.1371/journal.pone.0017571

Metag, J., \& Schäfer, M. S. (2018). Audience Segments in Environmental and Science Communication: Recent Findings and Future Perspectives. Environmental Communication, 12(8), 995-1004. https://doi.org/10.1080/17524032.2018.1521542

MORI. (2005). Science In Society: Findings from Qualitative and Quantitative Research.

Morrison, M., Duncan, R., Sherley, C., \& Parton, K. (2013). A comparison between attitudes to climate change in Australia and the United States. Australasian Journal of Environmental Management, 20(2), 87-100. https://doi.org/10.1080/14486563.2012.762946 
Morrison, Mark, Parton, Kevin, \& Hine, D. W. (2018). Increasing belief but issue fatigue: Changes in Australian Household Climate Change Segments between 2011 and 2016. PloS One, 13(6), e0197988. https://doi.org/10.1371/journal.pone.0197988

Neunhoeffer, F., \& Teubner, T. (2018). Between enthusiasm and refusal: A cluster analysis on consumer types and attitudes towards peer-to-peer sharing. Journal of Consumer Behaviour, 17(2), 221-236. https://doi.org/10.1002/cb.1706

Nisbet, M., \& Markowitz, E. M. (2014). Understanding public opinion in debates over biomedical research: Looking beyond political partisanship to focus on beliefs about science and society. PloS One, 9(2), e88473. https://doi.org/10.1371/journal.pone.0088473

Okamura, K. (2016). Dynamic development of public attitudes towards science policymaking. Public Understanding of Science (Bristol, England), 25(4), 465-479.

https://doi.org/10.1177/0963662515605420

OST, \& Wellcome Trust. (2000). Science and the Public. London.

Pullman, A., Chen, M. Y., Zou, D., Hives, B. A., \& Liu, Y. (2018). Researching multiple publics through latent profile analysis: Similarities and differences in science and technology attitudes in China, Japan, South Korea and the United States. Public Understanding of Science (Bristol, England), 963662518791902. https://doi.org/10.1177/0963662518791902

Research Councils UK. (2008). Public Attitudes to Science 2008: A Survey.

Runge, K. K., Brossard, D., \& Xenos, M. A. (2018). Protective Progressives to Distrustful Traditionalists: A Post Hoc Segmentation Method for Science Communication. Environmental Communication, 21(1), 1-23. https://doi.org/10.1080/17524032.2018.1513854

Schäfer, M. S., Füchslin, T., Metag, J., Kristiansen, S., \& Rauchfleisch, A. (2018). The different audiences of science communication: A segmentation analysis of the Swiss population's perceptions of science and their information and media use patterns. Public Understanding of Science (Bristol, England), 27(7), 836-856.

https://doi.org/10.1177/0963662517752886

Scheufele, D. A. (2018). Beyond the Choir?: The Need to Understand Multiple Publics for Science. Environmental Communication, 12(8), 1123-1126.

https://doi.org/10.1080/17524032.2018.1521543

Sweeney Research. (2011). Community Interest and Engagement with Science and Technology in Victoria 2011. Retrieved from http://science.sweeneyresearch.com.au/overview/

Swim, J. K., \& Geiger, N. (2015). From Alarmed to Dismissive of Climate Change: A Single Item Assessment of Individual Differences in Concern and Issue Involvement. Environmental Communication, 11(4), 568-586.

https://doi.org/10.1080/17524032.2017.1308409

Van der Linden, S. (2015). The social-psychological determinants of climate change risk perceptions: Towards a comprehensive model. Journal of Environmental Psychology, 41, 112-124. https://doi.org/10.1016/i.jenvp.2014.11.012 